\title{
TEM Study of Microstructure of Superhard c-BN/WC-Co Interface
}

\author{
H. H. Shen ${ }^{1,2}$, K. Sun ${ }^{2}$ X. Xiang ${ }^{1}$ and X. T. Zu ${ }^{1}$ \\ 1. School of Physical Electronics, University of Electronic Science and Technology of China, Chengdu \\ 610054, China \\ 2. Dept. of Materials Science and Engineering, University of Michigan, Ann Arbor, MI 48109, USA
}

\begin{abstract}
Advanced focused ion beam (FIB) technique, which has been well described by Mayer et al. [1], has been employed to prepare thin foils for transmission electron microscopy (TEM) investigation. It is a powerful tool for TEM sample preparation on nearly all kinds of materials. Especially, it can be served as an irreplaceable approach to prepare TEM specimens from some superhard materials that are difficult to be cut using regular methods such as diamond thin films [2]. The in situ FIB lift-out method mainly consists of extracting and transferring a wedge-shaped lamella, which is coated with a platinum $(\mathrm{Pt})$ thin layer to avoid $\mathrm{Ga}+$ irradiation damage. The lamella is transferred via an internal nanomanipulator and adhered to a TEM half-grid by Pt deposition. Finally, the lamella would be FIB milling to electron transparent and has a thickness of around $100 \mathrm{~nm}$.
\end{abstract}

Cemented tungsten carbide (WC-Co) is a hard material with excellent mechanical performance, of which improves its value in engineering industries as cutting tools [3]. Cubic-BN, associating with extreme hardness and resistance to wear, is addressed as the ideal coating material for WC-Co [4]. To ensure the practical applications of the multilayer superhard materials with distinct crystal structures, a good conjunction must be considered. Obviously, detailed knowledge is required to how the different hard materials connect and how the element diffuses at the interface in nano-scale. Here we report a TEM study on element distribution at the interface between two kinds of materials of WC-Co and c-BN. The TEM sample was successfully fabricating by utilizing the in-situ lift-out FIB method. The instrument used is a FEI dual platform Helios NanoLab 650 FIB. STEM and selected area electron diffraction (SAED) characterizations were conducted on a JEOL 2010 AEM working at $200 \mathrm{kV}$.

Figure 1(a) and (b) depict the FIB procedures to prepare cross-sectional TEM sample of WC-Co and c-BN. From Fig. 1(b), it indicates that an electron transparent area marked by dash line is obtained for TEM investigation. STEM bright field (ABF) and annular dark field (ADF) images are shown in Fig. 1(c) and 1(d), which are captured to present the features at the interface of WC-Co and c-BN. Energy dispersive X-ray spectroscopy (EDS) element mapping was performed to find out the element distributions at the interface. The color-mixed maps of two layers of c-BN and WC-Co are also in Fig. 2(h) and 2(i), from which Al-rich areas can be observed at the interface. The Al phase here functions as a binding material to make a good junction between the WC-Co substrate and the c-BN. It is clearly observed that many small particles of $\mathrm{W}$ and Co diffuse through the interface into the c-BN layer during the high temperature sintering and hot pressing processing.

In addition, SAED patterns were also performed aiming at confirming the crystal structures of the WC, $\mathrm{BN}$, Co and Al. Results indicate that structures of the WC, BN, Co and Al are hcp, fcc, fcc and fcc, respectively.

\section{References}

[1] J. Mayer et al., MRS Bull. 32 (2007), 400. 
[2] J.S. Park et al., J. Mater. Sci. 41 (2006), 4611.

[3] R.W. Armstrong et al., Materials 4 (2011), 1287.

[4] J. Echigoya et al., J. Mater. Sci. 32 (1997), 4693.
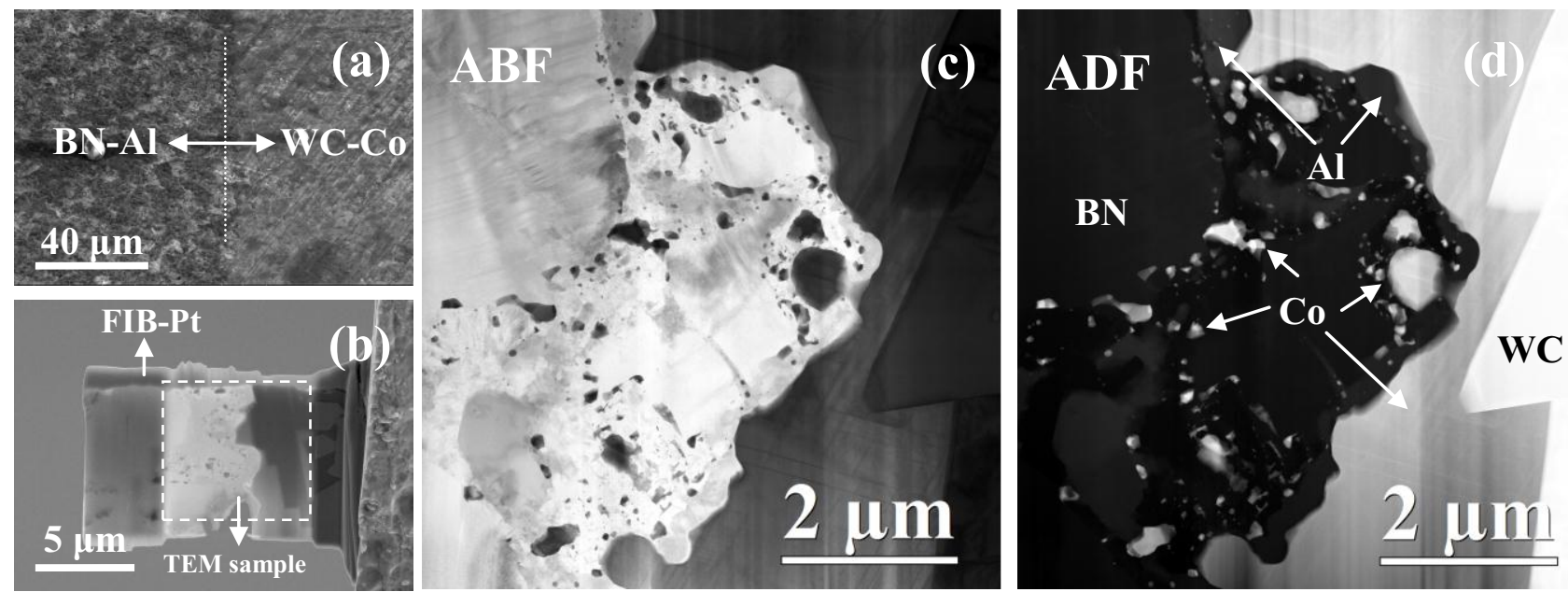

FIG. 1. SEM images of the c-BN/WC-Co interface (a) and FIB lift-out TEM specimen from the interface; (c) STEM BF and ADF (d) images taken from the in-situ lift-out specimen.
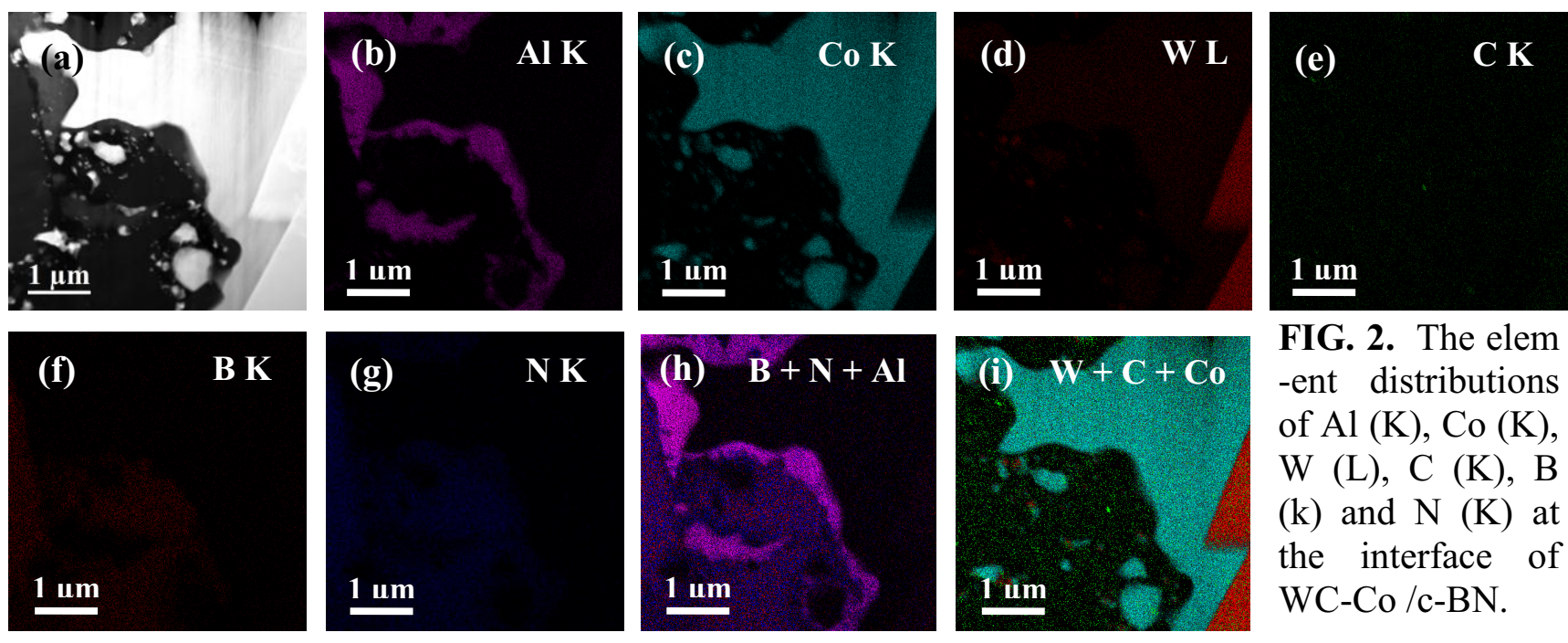

FIG. 2. The elem -ent distributions of $\mathrm{Al}(\mathrm{K}), \mathrm{Co}(\mathrm{K})$, $\mathrm{W}(\mathrm{L}), \mathrm{C}(\mathrm{K}), \mathrm{B}$ $(\mathrm{k})$ and $\mathrm{N}(\mathrm{K})$ at the interface of WC-Co /c-BN.
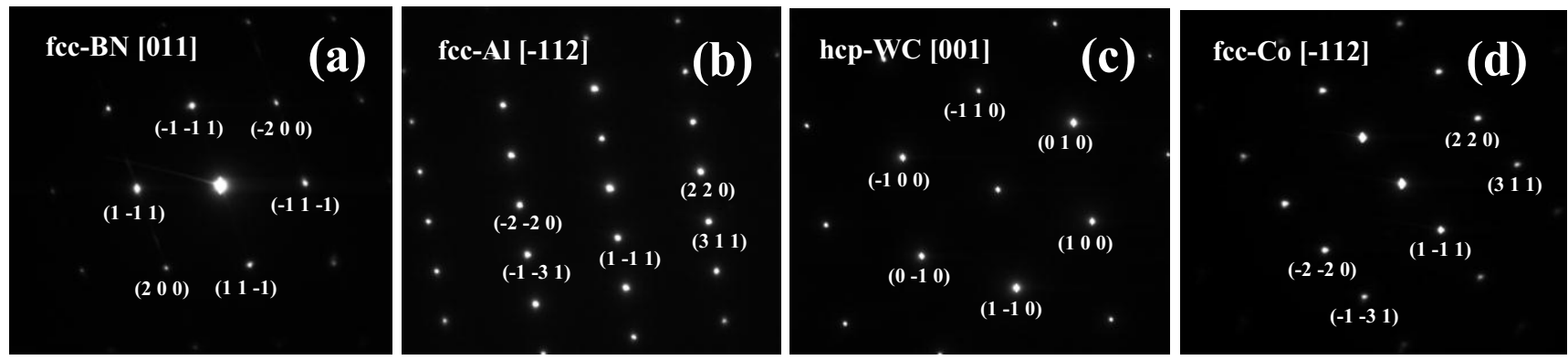

FIG. 3. SAED patterns of fcc-BN (a), fcc-Al (b), hcp-WC (c) and fcc-Co (d), respectively. 